\title{
Reação hansênica tipo dois em paciente multibacilar, forma Virchowiana, em vigência de tratamento: relato de caso
}

\author{
Erythema nodosum leprosum in multibacillary patient \\ during treatment: case report
}

\section{Luilson Geraldo Coelho Júnior ${ }^{1}$, Gabrielly Borges Machado², Talitha Araújo Faria ${ }^{3}$}

Coelho Júnior LG, Machado GB, Faria TA. Reação hansênica tipo dois em paciente multibacilar, forma Virchowiana, em vigência de tratamento: relato de caso / Leprosy reaction type two in multibacillary patient Lepromatous way in term of treatment: a case report. Rev Med (São Paulo). 2014 jul.-set.;94(3):197-200.

RESUMO: No Brasil, a hanseníase é um problema de saúde pública, devido sua alta incidência e ampla distribuição geográfica. É uma doença crônica causada pelo Mycobacterium leprae; acomete pele e nervos periféricos, podendo ocasionar lesões cutâneas, de aspecto variado: manchas, pápulas, placas, nódulos, infiltração difusa, dependendo da resposta imune do indivíduo. As reações hansênicas são fenômenos imuno inflamatórios agudos e subagudos podendo ocorrer antes, durante ou após o tratamento com a poliquimioterapia. As reações hansênicas são diagnosticadas através de exame físico geral, dermatológico e neurológico do paciente. O objetivo do presente trabalho é descrever um caso de reação hansênica tipo dois em paciente multibacilar, forma Virchowiana em vigência de tratamento.

Descritores: Hanseníase; Mycobacterium leprae; Hanseníase Virchowiana/diagnóstico.

\begin{abstract}
In Brazil, the leprosy is a public health problem, due to its high incidence and wide geographical distribution. It is a chronic disease caused by Mycobacterium leprae; affects skin and peripheral nerves, which can cause skin lesions, aspect varied: spots, papules, plaques, nodules, diffuse infiltration, depending on the immune response of the individual. The leprosy reactions are phenomena immune inflammatory acute and subacute and may occur before, during or after the treatment with multidrug therapy. The leprosy reactions are diagnosed through physical examination general, dermatological and neurological patient. The objective of this work is to describe a case of leprosy reaction type two in multibacillary patient, lepromatous in duration of treatment.
\end{abstract}

Keywords: Leprosy; Mycobacterium leprae; Leprosy, lepromatous/diagnosis.

1. Acadêmico de medicina da Faculdade Atenas, Paracatu, MG. E-mail: luilson-junior@hotmail.com.

2. Professora do Departamento de Clínica Médica da Faculdade Atenas, Paracatu, MG. E-mail: gabyborges100@hotmail.com.

3. Professora do Departamento de Pensamento Científico, Faculdade Atenas, Paractu, MG. E-mail: talithabio@yahoo.com.br.

Endereço para correspondência: Luilson Geraldo Coelho Júnior. Rua Bernardo Caparucho, 30, Ap 04. Bairro Bela Vista. Paracatu, MG. 


\section{INTRODUÇÃO} hanseníase é uma doença infectocontagiosa
de evolução crônica, granulomatosa, causada pelo Mycobacterium leprae, que acomete pele e nervos periféricos ${ }^{1}$. O Mycobacterium leprae é uma bactéria intracelular obrigatória, bacilo álcool-ácido resistente, de alta infectividade e baixa patogenicidade, podendo ocorrer em qualquer idade, raça, sexo ${ }^{1,2}$.

Após a Índia, o Brasil é o segundo país em número de casos no mundo; aproximadamente $94 \%$ dos casos novos diagnosticados nas Américas são notificados pelo Brasil $^{2}$. No município de Paracatu, a hanseníase tem alta prevalência, aproximadamnete 13,7/10.000 habitantes em $2003^{3}$.

A hanseníase constitui um problema de saúde pública, devido à possibilidade de gerar incapacidade física permanente, além de apresentar altos níveis endêmicos com uma variação na distribuição dos casos em todo o território nacional ${ }^{4}$. O que dificulta o controle epidemiológico desta doença.

A hanseníase pode ser classificada quanto às formas clínicas, exame histopatológico e resposta imune do paciente frente ao agente etiológico em forma indeterminada, tuberculóide, dimorfa e virchowiana ${ }^{5}$.

A Organização Mundial de Saúde (OMS) classificou os pacientes hansênicos em paucibacilares (PB) e multibacilares (MB) estabelecendo uma correlação entre diagnóstico e tratamento. A baciloscopia positiva classifica o caso como multibacilar, independentemente do número de lesões. Os paucibacilares possuem de uma a cinco lesões de pele e baciloscopia negativa, já os multibacilares possuem mais de cinco lesões de pele, independente do resultado da baciloscopia ${ }^{6}$.

Os surtos reacionais são episódios imuno inflamatórios que ocorrem durante a evolução da hanseníase, podendo acontecer antes do diagnóstico da doença, durante o tratamento ou após a alta. As reações hansênicas são classificadas em reação do Tipo um ou reação reversa e reação do Tipo dois ou eritema nodoso hansênico. Os surtos reacionais devem ser diagnosticados e tratados o mais precocemente possível ${ }^{7}$.

O tratamento da hanseníase engloba o uso de medicamentos na quimioterapia específica, as drogas usadas nos esquemas padronizados pela OMS são a rifampicina, dapsona e clofazimina. E nos surtos reacionais, reação tipo um, uso de prednisona e na reação tipo dois, uso de talidomida. Além disso, o tratamento visa prevenir as incapacidades físicas, bem como promover reabilitação física e psicossocial, do doente ${ }^{7-10}$.

O objetivo do presente trabalho é descrever um caso de reação hansênica tipo 2 em paciente multibacilar, forma Virchowiana, em vigência de tratamento.

\section{RELATO DE CASO}

Paciente masculino, 49 anos de idade, negro, casado, aposentado, natural de João Pinheiro -MG, residente no perímetro urbano de Paracatu-MG. Foi diagnosticado em Julho de 2014, em outro serviço de saúde, com Hanseníase, tendo sido notificado à Vigilância Epidemiológica e segundo a classificação operacional foi classificado como multibacilar, pois apresentou mais de cinco lesões de pele, além de baciloscopia positiva.

O diagnóstico foi estabelecido, tendo como base o quadro clínico: paciente com múltiplas lesões nodulares em tegumento de membros, face e tronco, que surgiram há cerca de um ano, com diminuição de sensibilidade térmica e dolorosa nas áreas lesionadas, associado a raspado dérmico, o qual evidenciou Índice Bacilar 4+/6+, resultado positivo; além de febre intermitente ao longo do primeiro ano de doença.

Após diagnóstico iniciou o tratamento com poliquimioterapia, fez uso de rifampicina dose mensal de $600 \mathrm{mg}$ (02 cápsulas de $300 \mathrm{mg}$ ) administração supervisionada; dapsona dose mensal $100 \mathrm{mg}$ supervisionada, uma dose diária 100mg autoadministrada; clofazimina dose mensal de 300mg (03 cápsulas de $100 \mathrm{mg}$ ) administração supervisionada e uma dose diária de 50mg autoadministrada. O paciente apresentou reação alérgica cutânea, urticariforme, à rifampicina, sendo que este medicamento foi retirado e foi administrado antihistamínico para tratamento da lesão urticariforme. O esquema terapêutico substitutivo à rifampicina instituído foi o uso de ofloxacina,dose mensal de $400 \mathrm{mg}$ supervisionada e dose diária de $400 \mathrm{mg}$ autoadministrada.

Durante o tratamento para hanseníase, no mês de agosto de 2014, o paciente apresentou reação hansênica tipo dois, cuja manifestação clínica mais frequente é o eritema nodoso hansênico, caracterizado por nódulos subcutâneos dolorosos, febre, artralgia, astenia e mal estar generalizado. Fez uso de talidomida $100 \mathrm{mg} /$ dia por 10 dias, sendo reavaliado após o fim do tratamento para o surto reacional, obtendo melhora, sendo suspensa a medicação.

Procurou o pronto socorro do Hospital Municipal de Paracatu, no mês de fevereiro de 2015, queixando-se de febre há 5 dias, dor abdominal, artralgia, astenia, dor abdominal e hiporexia. Apresentava nodulações dolorosas em membros inferiores. Paciente ficou em observação e foi internado, para avaliação do quadro.

No primeiro dia de internação, paciente apresentava febre $\left(39,9^{\circ} \mathrm{C}\right)$, pressão arterial $90 \times 60 \mathrm{mmHg}, \mathrm{FC}: 111 \mathrm{bpm}$, FR: 20irpm. Hemograma: hemácias: 3,90milhões/mm3, hematócrito: $33,9 \%$, hemoglobina: $11,1 \mathrm{~g} / \mathrm{dL}$, leucócitos e plaquetas normais. Ao exame físico dermatológico, observou-se a presença de nódulos subcutâneos dolorosos à palpação em membros inferiores e superiores, diminuição de sensibilidade térmica e dolorosa. Em exame físico abdominal, notou-se hepatomegalia $11 \mathrm{~cm}$ ao rebordo 
costal direito. Foi diagnosticado como reação hansênica do tipo dois ou eritema nodoso hansênico, tendo retomado o tratamento com talidomida. $\mathrm{O}$ paciente estava há cinco meses sem uso de talidomida, pois não apresentou reação hansênica durante esse período.

No segundo dia de internação, ao exame físico geral, paciente apresentava-se corado, hidratado, afebril $\left(36,7^{\circ} \mathrm{C}\right)$, FC $80 \mathrm{bpm}$, FR 22irpm, PA 120x80mmHG; realizou-se: Hemograma: hemácias: 4,23milhões/mm3, hematócrito: $35,0 \%$, hemoglobina: $12 \mathrm{~g} / \mathrm{dL}$, leucócitos e plaquetas normais. Os exames bioquímicos revelaram: sódio e potássio dentro da normalidade.

Ao exame físico dermatológico detectou-se: nódulos presentes em membros superiores e inferiores, porém notou-se diminuição do número de lesões, exame físico abdominal, notou-se hepatomegalia $11 \mathrm{~cm}$ ao rebordo costal direito. Ainda, o paciente apresentava-se com hiporexia, eliminações fisiológicas regulares. Foi mantido talidomida 400mg.

No terceiro dia de internação paciente apresentavase em bom estado geral, afebril, com diminuição da dor articular e regressão dos nódulos subcutâneos, apresentava eliminações fisiológicas regulares, sono reparador e aceitando a dieta.

Recebeu alta no quarto dia de internação e foi orientado a dar continuidade ao tratamento e procurar o serviço de saúde onde realiza o acompanhamento da doença. Foi feita retirada gradual da Talidomida, diminuindo a dose para $100 \mathrm{mg}$, tendo sido recomendado o uso por 10 dias e após completar esse esquema terapêutico cessou o uso.

\section{DISCUSSÃO}

A hanseníase é uma doença infecciosa crônica, que acomete pele e nervos periféricos, é uma doença com amplo espectro clínico é a resposta imune desencadeada pelo paciente frente ao Mycobacterium leprae que determina a forma que a doença se manifesta ${ }^{11}$.

Indivíduos que produzem resposta imunológica competente, do tipo Th1 desenvolvem formas paucibacilares, como a forma indeterminada e tuberculóide; já indivíduos que desenvolvem resposta imunológica do tipo Th2, manifestam formas multibacilares, como a forma Virchowiana e dimorfa ${ }^{2,12}$.

$\mathrm{Na}$ hanseníase tuberculóide, as manifestações clínicas estão relacionadas à exacerbação da resposta imune celular, há formação de granuloma bem definido, limitação das lesões e destruição completa dos bacilos. A hanseníase virchoviana se caracteriza por deficiência de resposta imune celular, excessiva multiplicação bacilar e disseminação da infecção para vísceras e tecido nervoso. É a forma de importância epidemiológica, pois os bacilos estão maciçamente presentes nas lesões cutâneas. A hanseníase dimorfa (HD), pode adquirir características tuberculóide (HDT) ou virchoviana (HDV), ou, simplesmente, permanecer como dimorfa (HDD), dependendo da potencialidade de resposta imune celular do hospedeiro ${ }^{13,14}$.

No caso descrito, o paciente apresentou a forma virchowiana da doença, decorrente da resposta imune do tipo Th2. O paciente apresentou infiltração difusa e progressiva da pele, o que a tornou xerótica, de aspecto apergaminhado, e acobreada. Surgiram pápulas, nódulos em face, tronco e membros superiores e inferiores. $O$ fígado foi acometido, gerando um quadro de hepatomegalia. Além da rarefação de pelos em membros e madarose, queda de pelos em cílios e supercilios ${ }^{1,12}$.

O eritema nodoso hansênico é uma reação inflamatória sistêmica relacionada à deposição de imunocomplexos. Sendo assim, mecanismos imunes humorais estão envolvidos na patogênese desse tipo de reação, que ocorre mais comumente em pacientes multibacilares ${ }^{15,16}$.

Durante a quimioterapia específica para hanseníase, o paciente apresentou reação hansênica tipo dois. É uma reação inflamatória aguda, sistêmica, em que há exacerbação da imunidade humoral, envolve a formação de imunocomplexos que circulam pelo sangue periférico e tem como manifestação clínica mais freqüente o Eritema Nodoso Hansênico5.

O paciente apresentava nódulos eritematosos dolorosos em membros superiores e inferiores e tronco; além de febre, artralgia e astenia, comuns no quadro clínico $^{12}$. O paciente fez uso de talidomida, medicamento recomendado pela organização mundial de saúde para tratar o eritema nodoso ${ }^{15}$. Não deve utilizar talidomida em mulheres em idade fértil e gestantes, devido efeito teratogênico na gravidez. Após iniciado o uso da talidomida, o paciente apresentou melhora, notando-se diminuição do número de lesões, melhora do aspecto da pele, diminuição da febre e artralgia.

A resposta terapêutica foi efetiva com o uso de talidomida, durante a reação hansênica, além disso, o esquema para pacientes MB preconizado pelo Ministério da Saúde foi mantido. Houve regressão parcial do quadro clínico, no quarto dia de tratamento o paciente não apresentou reação hansênica, recebendo alta, mostrando a rápida e efetiva resposta ao tratamento com uso da talidomida.

A correlação entre as formas clínicas e os estados reacionais é extremamente importante, haja vista, é o potencial imunogênico da bactéria que desencadeia as manifestações clínicas. Associado a isso, anamnese e exame físico, exames laboratoriais e baciloscopia ajudam a diagnosticar, a avaliar o prognóstico e a monitorar o tratamento. Logo, os estados reacionais constituem intercorrências no curso da doença e são as principais causas de incapacidades em pacientes portadores de 
hanseníase. A quimioterapia específica proporcionou novas perspectivas de cura para a hanseníase, a possibilidade de eliminação da doença reforçou a importância da prevenção, de modo a evitar as complicações clínicas decorrentes dos estados reacionais.

Conflito de interesse: Os autores declaram que não há conflito de interesses.

\section{REFERÊNCIAS}

1. Araújo MG. Hanseníase no Brasil. Rev Soc Bras Med Trop. 2003;36:373-82. http://dx.doi.org/10.1590/S003786822003000300010 .

2. Brasil. Ministério da Saúde. Secretaria de Políticas de Saúde. Departamento de Gestão de Políticas Estratégicas. Relatório de atividades da área técnica de dermatologia sanitária ano de 1999. Brasília; 1999.

3. Brasil. Ministério da Saúde. Secretaria de Políticas de Saúde. Hanseníase: atividades de controle e manual de procedimentos. Brasília; 2001.

4. Foss NT. Hanseníase: aspectos clínicos, imunológicos e terapêuticos. An Bras Dermatol. 1999;74:113-9.

5. Sousa ARD, et al. Hanseníase simulando erupção liquenóide: relato de caso e revisão de literatura. An Bras Dermatol. 2010;85(2):224-6. Disponível em: http://www.scielo.br/pdf/ $\mathrm{abd} / \mathrm{v} 85 \mathrm{n} 2 / 14 . \mathrm{pdf}$

6. Brasil. Ministério da Saúde. Secretaria de Políticas de Saúde. Guia para o controle da hanseníase. Brasília; 2002. p.18-20. Disponível em: http://bvsms.saude.gov.br/bvs/publicacoes/ guia_de_hanseniase.pdf.

7. Araujo MR. Hanseníase no Brasil. Rev Soc Bras Med Trop. 2003;36(3):373-82. http://dx.doi.org/10.1590/S003786822003000300010 .

8. Oliveira MLWDR. Cura da hanseníase: magnitude das recidivas no Brasil, estudo de coorte de diferentes esquemas terapêuticos e fatores de risco. Rio de Janeiro: Universidade Federal do Rio de Janeiro; 1996.

9. Brasil. Ministério da Saúde. Portaria No 1073/GM de 26 de setembro de 2000. D.O.U., seção I, 188-E, p.18, 28 de setembro de 2000 .

10. Nery JA, Vieira LMM, Matos HJ, Gallo MEN, Sarno EN. Reactional states in multibacillary Hansen disease patients during multidrug therapy. Rev Inst Med Trop São Paulo. 1999;40:363-70. http://dx.doi.org/10.1590/S003646651998000600005.

11. Yoder LD, Guerra IE. Hansen's disease. A guide to management in the United States. Hansen's Disease Foundation; 2001.

12. Talhari S, Neves RG. Dermatologia tropical-Hanseníase. Manaus: Gráfica Tropical; 1997.

13. Stefani MM, Martelli CM, Gillis TP, Krahenbuhl JL. In situ type 1 cytokine gene expression and mechanisms associated with early leprosy progression. J Infect Dis. 2003;188:102431. doi: $10.1086 / 378410$.

14. Goulart IMB, Penna GO, Cunha G. Imunopatologia da hanseníase: a complexidade da resposta imune do hospedeiro ao Mycobacterium leprae. Rev Soc Bras Med Trop. 2002;35:365-75. http://dx.doi.org/10.1590/S003786822002000400014 .

15. Pocaterra L, et al. Clinical course of erythema nodosum leprosum: an 11-year cohort study in Hyderabad, India. Am J Trop Med Hyg. 2006;74:868-79. Available from: http:// www.ajtmh.org/content/74/5/868.long.

16. Naafs B. Treatment of leprosy: science or politics? Trop Med Int Health. 2006;11:268-78. doi: 10.1111/j.13653156.2006.01561.x. 